Prefacio

\title{
Interacción entre la Arqueología y las Ciencias de la Tierra: Nuevos avances en Geoarqueología
}

\author{
Ángel Carrancho, Catriel Greco
}

\begin{abstract}
Ángel Garrancho
acarrancho@ubu.es

Área de Prehistoria, Departamento de Historia, Geografía y Comunicación, Universidad de Burgos. Plaza Misael Bañuelos S/N. 09001 Burgos, España.
\end{abstract}

\section{Catriel Greco}

CONICET, Departamento de Geología, Universidad Nacional de San Luis. Avenida Ejército de Los Andes 950, D5700HHW, San Luis, Argentina.

BOL. SOC. GEOL. MEX. 2019

VOL. 71 NO. 2

P. $\mathrm{v}-$ viii

http://dx.doi.org/10.18268/BSGM2019v7 1n2p2
Los contextos arqueológicos son generalmente complejos y su estudio requiere de la aplicación combinada de diferentes disciplinas científicas con técnicas de alta resolución. En los últimos años, estamos presenciando cómo la geoarqueología, una disciplina a caballo entre las Ciencias de la Tierra, la Arqueología y la Historia, está aportando valiosa información para la reconstrucción de procesos de formación en yacimientos arqueológicos y dinámicas paleoambientales en general. El número de publicaciones internacionales sobre geoarqueología ha aumentado significativamente en las últimas dos décadas, lo que evidencia la firme apuesta en investigación por la aplicación de métodos de las Ciencias de la Tierra a la solución de problemas arqueológicos. Los arqueólogos son conscientes que los contextos estratigráficos donde se encuentran los restos culturales contienen información de gran valor para reconstruir las actividades en el pasado. Los geólogos, edafólogos, estratígrafos y otra suerte de expertos que podríamos integrar en el ámbito de las Geociencias, ven en los yacimientos y materiales arqueológicos laboratorios idóneos donde testar la aplicabilidad de múltiples técnicas. Esa sinergia entre el elemento humano y su contexto geológico es donde la geoarqueología hunde sus raíces pero, a pesar de su enorme potencial, todavía es un ámbito por explorar.

$\mathrm{El}$ presente volumen contiene numerosas contribuciones aplicadas a contextos o problemáticas geoarqueológicas de diversa edad y naturaleza. Se incluyen trabajos de geomorfología e hidrología (Heider $e t$ al.), una aplicación informática aplicada a la edafología (Palma-López y Bautista), quimioestratigrafía (Iriarte et al.), análisis geomorfológicos y cartográficos (Caria y Gómez-Augier, Sampietro-Vattuone y Peña-Monné), topografia, cartografia, mineralogía y arqueología (dos artículos de Díaz-Acha et al.), fluorescencia y difracción de rayos X, espectrorradiometría y micromorfología (Blancas et al.), arqueomagnetismo, espectroscopía Raman y arqueología experimental (Carrancho et al.), petrología y micromorfología (Castañeda et al.), arqueomagnetismo (Olay et al., Cervantez-Solano et al., Cifuentes-Nava et al., Morales et al., Soler-Arechalde et al.), pedología, magnetismo y micromorfología de suelos (Cruz-y-Cruz et al.), datación por radiocarbono y microscopía electrónica de barrido (Gómez González et al.), estudios isotópicos (Pérez-Crespo et al.), espectrometría de masas y difracción de rayos $\mathrm{X}$ (Puy-Alquiza et al.). 
Este volumen comienza con Heider et al., quienes aplican análisis geomorfológicos e hidrológicos de paisajes de dunas en el centro de Argentina para reconstruir su uso como eco-refugios por grupos prehispánicos. La aplicación de estos análisis junto con otras evidencias arqueológicas y etnográficas, constituyen la base de un modelo de ocupación del espacio donde la gestión del agua es el factor clave en un entorno árido.

Palma-López y Bautista presentan una novedosa aplicación informática que incorpora datos edafológicos de suelos mayas. La aplicación incluye la descripción de los suelos mayas, fotografias, un mapa de la clasificación maya de suelo en el estado mexicano de Yucatán y se explica su estructura y funcionamiento en un formato didáctico y manejable.

El estudio de Iriarte et al. representa un ejemplo de transdisciplinariedad integrando análisis estratigráficos, sedimentológicos y geoquímicos en un sondeo del yacimiento Neolítico de Los Cascajos, en la península ibérica. Los autores reconstruyen la evolución paleoambiental del área a lo largo del Holoceno, asociando la ocupación antrópica con fases más húmedas que la actual e identificando fases de aridificación posteriores en el registro sedimentario.

Caria y Gómez-Augier investigan mediante análisis geomorfológicos, SIG y arqueológicos los patrones de uso del espacio durante la época prehispánica en el sector septentrional de las tierras bajas de Tucumán, Argentina. Evaluar los procesos morfodinámicos a diferentes escalas es útil no sólo para reconstruir los patrones de ocupación espacial sino también para evaluar su incidencia en la localización y preservación del registro material.

Díaz-Acha et al. presentan dos interesantes estudios geoarqueológicos aplicados a las minas neolíticas de variscita de Gavà, en el noreste de la península ibérica, que son las minas subterráneas más antiguas de Europa occidental. En la primera contribución, Díaz-Acha et al. (I) establecen criterios sobre la exploración y explotación de la variscita. Mediante cortes y mapas, datos geológicos, mineralógicos y arqueológicos, los autores describen cómo se forma y dónde se localiza la variscita y reconstruyen la dinámica de uso y explotación del complejo minero. En su segunda contribución, Díaz-Acha et al. (II) estudian el contexto geológico y reconstruyen hasta tres procesos de mineralización de hierro, así como describen las estrategias de explotación minera activas en el sitio también en épocas históricas.

El estudio de Blancas et al. sobre el barrio de Tlajinga, Teotihuacán (México), constituye otro caso de estudio enfocado desde una perspectiva multianalítica. La combinación de análisis de fluorescencia y difracción de rayos $\mathrm{X}$, complementados con resultados de percepción remota, espectrorradiometría y micromorfología, permiten caracterizar la composición elemental de las partículas del suelo y diversos materiales de interés arqueológico.

Otro enfoque geoarqueológico interesante lo ofrecen Carrancho et al., quienes caracterizan las alternaciones mineralógicas producidas por el fuego en calizas combinando arqueología experimental, arqueomagnetismo, propiedades magnéticas y espectroscopía Raman. La aplicación conjunta de estos métodos en la caracterización mineralógica del material refleja su complementariedad y viabilidad para ser aplicadas a contextos arqueológicos potencialmente afectados por fuego.

Castañeda et al. reconstruyen la cadena operatoria de la producción cerámica Xajay (México) mediante análisis estadísticos de resultados petrológicos y micromorfológicos. Los resultados les han permitido plantear dinámicas de explotación y preparación del barro para la elaboración cerámica demostrando que la mayoría de la producción era local mientras que una pequeña parte se producía en otras regiones. 
El estudio arqueomagnético de Olay et al. sobre fragmentos cerámicos y restos de bajareque quemado del Valle de Colima (México) es otro ejemplo interesante de aplicación geoarqueológica de esta técnica cronométrica. Los autores fechan dentro del período Clásico mesoamericano una serie de vestigios con el fin de elaborar un marco cronológico de referencia para este ámbito.

Cervantes-Solano et al. presentan resultados de datación arqueomagnética de flujos de lava del volcán Xitle, al sur del Valle de México. Los resultados les permiten identificar dos periodos eruptivos, siendo el segundo compatible cronológicamente con el abandono paulatino del sitio arqueológico pre-Clásico de Cuicuilco. Precisar esta cronología es muy importante para entender los grandes cambios en el contexto económico y social regional que sucedieron a estos acontecimientos volcánicos.

En una línea de trabajo similar, Cifuentes-Nava et al. realizaron dataciones de arqueomagnetismo de cinco flujos de lava del volcán Ceboruco, Jalisco (México). La aplicación de este método mediante el modelo de variación del campo magnético SHA.DIF.14K y la comparación con edades radiométricas previamente reportadas, permite establecer con mayor certeza las edades de los emplazamientos y discutir la confiabilidad del método arqueomagnético como herramienta para fechar erupciones volcánicas recientes.

Cruz-y-Cruz et al. evalúan las características de tres perfiles de secuencias pedosedimentarias formadas durante el Holoceno tardío en La Playa, Sonora (NW de México). Tomando en cuenta estudios interdisciplinarios de distintos rasgos pedológicos, llegan a conclusiones de gran interés sobre la influencia que las actividades de subsistencia humana diaria tuvieron en la configuración de los suelos antiguos y modernos.

También con un caso de estudio en México, Gómez González et al. realizaron dataciones de radiocarbono de un petate elaborado con fibras duras y una mortaja de algodón de un fardo mortuorio de Zimapán, Hidalgo. Asimismo, utilizaron microscopía electrónica de barrido para determinar el estado de conservación de la pieza y los efectos de aplicación de un tratamiento de conservación innovador, del cual también evalúan como afecta envejeciendo el contenido de radiocarbono en el material tratado.

Morales et al. presentan los primeros resultados de determinaciones de intensidad magnética en fragmentos de cerámica de sociedades indígenas de Uruguay. El muestreo incluyó material de nueve sitios arqueológicos distribuidos por todo el país y las edades estimadas a partir del arqueomagnetismo son coherentes con las cronologías obtenidas por ${ }^{14} \mathrm{C}$ de los mismos sitios.

Pérez-Crespo et al. realizan estudios paleoambientales para sitios del Pleistoceno tardío de México. A partir de la revisión de datos previamente publicados, analizan las relaciones isotópicas de carbono y oxígeno presentes en el esmalte dental de la mastofauna hallada en sitios con presencia humana. Encuentran que había mayor humedad que en la actualidad, permitiendo el establecimiento de especies vegetales y animales que los primeros pobladores pudieron aprovechar.

Puy-Alquiza et al. estudian las características estructurales, físicas, mecánicas y químicas de adobes procedentes de haciendas mineras de los Siglos XVII y XVIII de Guanajuato. Esto apunta a crear una base de conocimiento sobre el material, tanto para evaluar su seguridad como para contribuir a la conservación de la arquitectura del adobe. El análisis de las muestras implicó el uso de varias técnicas, como Espectrometría de Masas de Plasma Acoplado Inductivamente, Difracción de Rayos X, Microscopía Electrónica de Barrido y ensayos mecánicos.

Sampietro-Vattuone y Peña-Monné presentan una elaborada propuesta metodológica para el estudio geoarqueológico de cambios ambienta- 
les en medios áridos y semiáridos subtropicales, a partir de casos de estudio de áreas arqueológicas, tanto de Argentina como de España, caracterizadas por una economía agropastoril preindustrial. La realización de mapas y perfiles geomorfológicos y la elaboración de modelos evolutivos de la reconstrucción paleoambiental son explicados con detalles, proveyendo a los lectores de una excelente guía para la planificación de un trabajo geoarqueológico.

Por último, el trabajo de Soler-Arechalde et al. es una valiosa compilación a modo de catálogo de resultados de arqueomagnetismo confiables para el centro y sur de México, o sea, la porción central y norte de Mesoamérica. Asimismo, se suma una gran cantidad de nuevos resultados, lo que en conjunto permite presentar una nueva curva de variación paleosecular para la región.

Deseamos que los artículos compilados en este volumen proporcionen una lectura agradable a los lectores y sobre todo, estimule profundas discusiones científicas. Para nosotros las contribuciones aquí presentadas han impulsado nuestra compresión de la Geoarqueología a la vez que también han abierto preguntas y desafios interesantes de cara al futuro. 